\title{
Super-Planckian far-field radiative heat transfer
}

\author{
V. Fernández-Hurtado, ${ }^{1}$ A. I. Fernández-Domínguez, ${ }^{1}$ J. Feist, ${ }^{1}$ F. J. García-Vidal,,${ }^{1,2}$ and J. C. Cuevas ${ }^{1,3}$ \\ ${ }^{1}$ Departamento de Física Teórica de la Materia Condensada and Condensed Matter Physics Center (IFIMAC), \\ Universidad Autónoma de Madrid, E-28049 Madrid, Spain \\ ${ }^{2}$ Donostia International Physics Center (DIPC), Donostia/San Sebastián 20018, Spain \\ ${ }^{3}$ Department of Physics, University of Konstanz, D-78457 Konstanz, Germany
}

(Received 21 August 2017; revised manuscript received 21 December 2017; published 8 January 2018)

\begin{abstract}
We present here a theoretical analysis that demonstrates that the far-field radiative heat transfer between objects with dimensions smaller than the thermal wavelength can overcome the Planckian limit by orders of magnitude. To guide the search for super-Planckian far-field radiative heat transfer, we make use of the theory of fluctuational electrodynamics and derive a relation between the far-field radiative heat transfer and the directional absorption efficiency of the objects involved. Guided by this relation, and making use of state-of-the-art numerical simulations, we show that the far-field radiative heat transfer between highly anisotropic objects can largely overcome the black-body limit when some of their dimensions are smaller than the thermal wavelength. In particular, we illustrate this phenomenon in the case of suspended pads made of polar dielectrics like SiN or $\mathrm{SiO}_{2}$. These structures are widely used to measure the thermal transport through nanowires and low-dimensional systems and can be employed to test our predictions. Our work illustrates the dramatic failure of the classical theory to predict the far-field radiative heat transfer between micro- and nanodevices.
\end{abstract}

DOI: 10.1103/PhysRevB.97.045408

\section{INTRODUCTION}

Thermal radiation is a ubiquitous physical phenomenon and its understanding is critical for many different technologies [1]. This understanding is still largely based on Planck's law for black bodies [2]. In particular, this law sets an upper limit for the radiative heat transfer (RHT) between bodies at different temperatures. However, this law has known limitations. One of them is its inability to describe the RHT between objects separated by distances smaller than the thermal wavelength ( $\lambda_{\mathrm{Th}} \approx 10 \mu \mathrm{m}$ at room temperature) [3-5]. As predicted in the 1970s [6] within the theory of fluctuational electrodynamics (FE) [7], RHT in this near-field regime is dominated by evanescent waves and the Planckian limit can be far surpassed by bringing objects sufficiently close. The experimental verification of this prediction in recent years has boosted the field of thermal radiation [8-21] and has triggered the hope that near-field RHT can have an impact in different thermal nanotechnologies $[4,5]$.

As already acknowledged by Planck in his seminal work [2], another limitation of his law is related to the description of RHT between objects with dimensions smaller than $\lambda_{\text {Th }}$. In this case, Planck's law, which is based on ray optics, is expected to fail even in the far-field regime, where separations are larger than $\lambda_{\mathrm{Th}}$. Thus, one may wonder whether the Planckian limit can also be overcome in the far-field regime, something that is not possible with extended (infinite) objects [22]. It is known that the emissivity of a finite object can be greater than 1 at certain frequencies [23,24], but that is not enough to emit more than a black body. In fact, only a modest super-Planckian thermal emission has been predicted in rather academic situations [25,26], and it has never been observed. For instance, Ref. [27] reports one of the few experiments exploring the thermal radiation of an object smaller than $\lambda_{\mathrm{Th}}$. In that work, the thermalization of an optical fiber was investigated and Planck's law was shown to incorrectly describe the thermal emission, but the fiber was actually found to emit much less than a corresponding black body, i.e., no super-Planckian thermal emission was observed. In this work, we are interested not in the emission of a single object, but in the case of radiative heat transfer between two objects. In this context, there are neither theoretical proposals nor observations of super-Planckian farfield RHT. This fact is mainly due to the lack, until recently, of numerical techniques able to describe the RHT between objects of arbitrary size and shape that can, in turn, guide the design of appropriate experiments. It is also worth stressing that the problem of whether the Planckian limit can be overcome in the far-field regime is not only of fundamental interest, but it is also of great practical importance because Planck's law continues to be used to obtain an upper bound for far-field RHT between microdevices [28-33].

The goal of this work is to demonstrate that Planck's law can fail dramatically when describing the far-field RHT between finite objects and, more importantly, that the Planckian limit can be greatly overcome in the far-field regime. For this purpose, we have combined state-of-the-art numerical simulations within $\mathrm{FE}$ with analytical insight provided by a general relation derived here between the far-field RHT between finite objects and their directional absorption efficiencies. Guided by this relation, we show that the far-field RHT between highly anisotropic systems can greatly overcome the Planckian limit when some of their dimensions are smaller than $\lambda_{\text {Th }}$. This exploits that due to its inherent directionality, super-Planckian RHT can occur even if the involved objects are not superPlanckian emitters. In particular, we illustrate this phenomenon with the analysis of the RHT between micrometer-sized 
suspended pads made of polar dielectrics $\left(\mathrm{SiN}\right.$ and $\left.\mathrm{SiO}_{2}\right)$. To be precise, we show that when the thickness of these pads is much smaller than $\lambda_{\mathrm{Th}}$, the far-field radiative thermal conductance at room temperature can be several orders of magnitude higher than that between black bodies of the same dimensions. Let us stress that this type of dielectric pads are widely used in the context of thermal transport measurements of nanowires and low-dimensional systems [28-33], and they thus constitute an available technology with which our predictions can be quantitatively tested.

The rest of the paper is organized as follows. In Sec. II, we derive several analytical formulas relating the far-field RHT between finite objects and the directional absorption efficiencies. Section III is devoted to illustrate why it is so difficult to overcome the Planckian limit in the far-field regime. For this purpose, we present in this section results for the far-field RHT between optically isotropic systems like spheres and cubes. In Sec. IV, we demonstrate the possibility to greatly overcome the Planckian limit in the far field by exploring the RHT between parallelepipeds made of polar dielectrics ( $\mathrm{SiN}$ and $\mathrm{SiO}_{2}$ ). Then, in Sec. V, we illustrate the super-Planckian far-field RHT in suspended pads made of dielectric materials, where we show in particular that our predictions can be quantitatively tested with existent technology. We conclude the paper in Sec. VI with a brief summary of the main conclusions of our work. Additionally, we have included three appendixes where we present the dielectric functions used in our calculations (Appendix A), provide additional results for $\mathrm{SiO}_{2}$ (Appendix B), and report the expressions of the view factors for the different geometries explored in this work (Appendix C).

\section{RELATION BETWEEN FAR-FIELD RADIATIVE HEAT TRANSFER AND ABSORPTION EFFICIENCIES}

In principle, the theory of FE provides a general framework to describe the RHT between arbitrary objects both in the near-field and in the far-field regime [7]. So, in this sense, one may be tempted to think that the search for super-Planckian far-field RHT should be straightforward. However, in practice, one encounters several problems. First of all, there is an infinite number of possibilities in terms of materials, shapes, and sizes of the bodies. More importantly, until very recently, there were no numerical methods capable of describing the RHT between objects of arbitrary size and shape, and this actually continues to be a very important practical problem that is often insurmountable. For this reason, it would be highly desirable to have, for instance, a relation between the far-field RHT of objects of arbitrary size and shape and their radiation absorption properties, something that has been widely studied over the years [23]. The goal of this section is to present such a relation, which will turn out to be extremely useful in our quest for super-Planckian far-field RHT.

In what follows, we derive a relation between the far-field RHT between two objects and their directional absorption efficiencies. This result was obtained with the help of the thermal discrete dipole approximation (TDDA) put forward by some of us in Ref. [34], see also Refs. [35,36]. This approach allows us to compute the RHT between finite objects of arbitrary size and shape within the framework of FE. The full derivation is actually quite cumbersome. For this reason, we shall show in some detail how this result can be obtained in the limit of very small objects and then, we shall indicate how it can be extended to objects of arbitrary size. So, let us first consider the RHT between two small objects that can be approximated by (electrical) dipolar particles. In this case, the power exchanged by them in the form of thermal radiation is given by Eq. (35) in Ref. [34]. That equation tells us that the net power exchanged by two identical dipoles at temperatures $T_{1}$ and $T_{2}$ and located at positions $\mathbf{r}_{1}$ and $\mathbf{r}_{2}$ such that their separation $d=\left|\mathbf{r}_{2}-\mathbf{r}_{1}\right|$ is much larger than the thermal wavelength is given by

$$
\begin{aligned}
P= & 8 \pi^{2} \int_{0}^{\infty} k_{0}^{2} \operatorname{Tr}\left\{\hat{G} \hat{\chi} \hat{G}^{\dagger} \hat{\chi}\right\} \\
& \times\left[I_{\mathrm{BB}}\left(\omega, T_{1}\right)-I_{\mathrm{BB}}\left(\omega, T_{2}\right)\right] d \omega .
\end{aligned}
$$

Here, $\omega$ is the radiation frequency, $k_{0}=\omega / c, c$ being the speed of light in vacuum, and $I_{\mathrm{BB}}(\omega, T)$ is the Planck distribution function given by

$$
I_{\mathrm{BB}}(\omega, T)=\frac{\omega^{2}}{4 \pi^{3} c^{2}} \frac{\hbar \omega}{\exp \left(\hbar \omega / k_{\mathrm{B}} T\right)-1},
$$

where $\hbar$ is Planck's constant, $k_{\mathrm{B}}$ is Boltzmann's constant, and $T$ is the temperature. On the other hand, the $(3 \times 3)$ matrix $\hat{\chi}$ is given by [34]

$$
\hat{\chi}=\frac{1}{2 i}\left(\hat{\alpha}-\hat{\alpha}^{\dagger}\right)-\frac{k_{0}^{3}}{6 \pi} \hat{\alpha}^{\dagger} \hat{\alpha},
$$

where $\hat{\alpha}$ is the polarizability tensor of the particles. Finally, $\hat{G}$ is the dyadic Green tensor connecting the two dipoles, which in the far-field limit is given by $\hat{G}=e^{i k_{0} d}[1-\hat{\mathbf{r}} \otimes \hat{\mathbf{r}} /(4 \pi d)]$, where $\hat{\mathbf{r}}=\left(\mathbf{r}_{2}-\mathbf{r}_{1}\right) / d$ and $\otimes$ denotes the exterior product. The result of Eq. (1) is valid for dipolar particles of arbitrary shape and made of materials with an arbitrary dielectric tensor. Let us focus now on the case of optically isotropic materials and particles with a scalar polarizability tensor, like spheres or cubes, such that $\hat{\chi}=\chi \hat{1}$. In this case, one can show that the absorption cross section, which in this case is independent of the direction and of the light polarization, is given by $\sigma_{\mathrm{abs}}(\omega)=$ $k_{0} \chi(\omega)$ [34]. Thus it is easy to show that Eq. (1) reduces to

$$
P=\pi A F_{12} \int_{0}^{\infty} Q^{2}(\omega)\left[I_{\mathrm{BB}}\left(\omega, T_{1}\right)-I_{\mathrm{BB}}\left(\omega, T_{2}\right)\right] d \omega,
$$

where $A$ is the area of the bodies, $F_{12}$ is a geometrical view factor, and $Q(\omega)$ is the absorption efficiency defined as $Q(\omega)=$ $\sigma_{\mathrm{abs}}(\omega) / \sigma_{\text {geo }}$, where $\sigma_{\text {geo }}$ is the geometrical or physical cross section. For isotropic particles, the absorption cross section is given by [34]

$$
\sigma_{\text {abs }}(\omega)=\frac{k_{0} \operatorname{Im}\left\{\alpha_{0}(\omega)\right\}}{\left|1-i k_{0}^{3} \alpha_{0}(\omega) /(6 \pi)\right|^{2}},
$$

where $\alpha_{0}(\omega)=3 V[\epsilon(\omega)-1] /[\epsilon(\omega)+2]$ is the quasistatic polarizability, $\epsilon(\omega)$ being the frequency-dependent dielectric function of the material, and $V$ is the volume of the particle. In the case of spherical particles, we have that

$$
A=4 \pi R^{2}, F_{12}=\frac{R^{2}}{4 d^{2}}, \sigma_{\mathrm{geo}}=\pi R^{2}, V=\frac{4}{3} \pi R^{3},
$$


where $R$ is the particle radius. In the case of cubic particles, we have

$$
A=L^{2}, F_{12}=\frac{L^{2}}{\pi d^{2}}, \sigma_{\text {geo }}=L^{2}, V=L^{3},
$$

where $L$ is the cube side. Let us recall that for black bodies $Q(\omega)=1$ for all frequencies and Eq. (4) reduces to the Stefan-Boltzmann law [1]: $P_{\mathrm{BB}}=\sigma A F_{12}\left(T_{1}^{4}-T_{2}^{4}\right)$, where $\sigma=5.67 \times 10^{-8} \mathrm{~W} /\left(\mathrm{m}^{2} \mathrm{~K}^{4}\right)$.

As we shall show later in this work, we need a certain degree of anisotropy in the shape of the bodies to achieve the super-Planckian far-field RHT. For this reason, we now consider the simplest case of anisotropic dipolar particles, namely rectangular parallelepipeds of sides $L_{x}, L_{y}$, and $L_{z}$. Here, we assume that the $z$ axis is oriented along the line joining the two dipolar particles. In this case, the polarizability tensor adopts the diagonal form $\hat{\alpha}=\operatorname{diag}\left(\alpha_{x}, \alpha_{y}, \alpha_{z}\right)$, which from Eq. (3) implies that $\hat{\chi}=\operatorname{diag}\left(\chi_{x}, \chi_{y}, \chi_{z}\right)$ with $\chi_{j}=$ $\operatorname{Im}\left\{\alpha_{j}\right\}-\left(k_{0}^{3} / 6 \pi\right)\left|\alpha_{j}\right|^{2}$ and $j=x, y, z$. Thus, using Eq. (1), it is straightforward to show that the net power exchanged by these two dipolar particles is given by

$$
\begin{aligned}
P= & \pi A F_{12} \int_{0}^{\infty} \frac{1}{2}\left(Q_{x}^{2}(\omega)+Q_{y}^{2}(\omega)\right) \\
& \times\left[I_{\mathrm{BB}}\left(\omega, T_{1}\right)-I_{\mathrm{BB}}\left(\omega, T_{2}\right)\right] d \omega,
\end{aligned}
$$

where $A=L_{x} L_{y}, F_{12}=L_{x} L_{y} /\left(\pi d^{2}\right)$, and $Q_{x, y}(\omega)$ are the absorption efficiencies for a plane wave impinging in the parallelepiped along the $z$ direction and with linear polarization along the $x$ and $y$ directions, respectively. These efficiencies are defined as $Q_{j}(\omega)=\sigma_{\mathrm{abs}, j}(\omega) / \sigma_{\mathrm{geo}}(j=x, y)$, where $\sigma_{\mathrm{geo}}=$ $L_{x} L_{y}$ and $\sigma_{\mathrm{abs}, j}=k_{0} \chi_{j}$. Finally, the different components of the polarizability tensor are given by [34]

$$
\alpha_{j}(\omega)=\frac{V}{\frac{1}{\epsilon(\omega)-1}+\Lambda_{j}-i V \frac{k_{0}^{3}}{6 \pi}},
$$

with $V=L_{x} L_{y} L_{z}$ and

$$
\Lambda_{j}=\frac{2}{\pi} \arctan \left(\frac{V}{L_{j}^{2} \sqrt{L_{x}^{2}+L_{y}^{2}+L_{z}^{2}}}\right) .
$$

Again, the black-body limit is obtained by assuming that $Q_{x, y}(\omega)=1$ for all frequencies.

Equations (4) and (8) can be generalized to the case of objects of arbitrary size employing the full TDDA formulation detailed in Sec. IV of [34]. In particular, the starting point is Eq. (56) in that reference, together with the far-field approximation of Eq. (58). The results of Eq. (4), for isotropic objects, and Eq. (8), for parallelepipeds, are recovered when the gap is much larger than both the thermal wavelength and the characteristic dimensions of the objects. In the generalized formulas, the absorption efficiencies correspond to plane waves with normal incidence (along the line joining the two objects) and with a given polarization. It is important to stress that the corresponding absorption cross sections are no longer given by the analytical formulas above for dipolar particles, but they have to be calculated numerically, something that we have done with either the DDA method [34] or with COMSOL MULTIPHYSICS. The corresponding view factors for finite objects are taken from Ref. [1], except for spheres, which are taken from Ref. [37] (see Appendix C). In the following sections we shall test those formulas for bodies of arbitrary size against numerically exact calculations carried out with the TDDA method [34] and SCUFF-EM [38,39]. In all cases, our numerical results confirm the validity of these formulas relating the far-field RHT with the absorption efficiencies.

To conclude this section, let us say that Eq. (4) has the expected form from the expression of thermal emission of a sphere [7,23,25], where $Q(\omega)$ is independent of the direction and the polarization. However, to our knowledge, this result has not been reported in the literature for bodies of arbitrary size and its generalization to anisotropic bodies, for instance, Eq. (8), is by no means trivial. As we shall see in the following section, these expressions provide very useful guidelines for the search of super-Planckian far-field RHT.

\section{WHY IS IT SO DIFFICULT TO OVERCOME THE PLANCKIAN LIMIT IN THE FAR FIELD?}

As explained in the introduction, super-Planckian far-field RHT has never been reported. Thus, we find instructive to devote this section to explain why this is a nontrivial phenomenon.

Equations (4) and (8) derived in the previous section seem to provide a straightforward recipe to overcome the Planckian limit in the far-field regime. Since the absorption efficiency of a finite object can be larger than unity [23], those equations suggest that super-Planckian far-field RHT might be possible if we find the right combination of material and object shape that leads to resonant absorption close to the maximum of Planck's distribution at a given temperature. This appealing idea is, however, not easy to realize in practice, as we proceed to illustrate. For simplicity, let us first study the far-field RHT between two identical spheres of arbitrary radius. In Fig. 1, we show the results for the absorption efficiency and the far-field RHT for spheres made of a metal (Au) and two polar dielectrics $\left(\mathrm{SiO}_{2}\right.$ and $\left.\mathrm{SiN}\right)$ with radii ranging from $100 \mathrm{~nm}$ to $50 \mu \mathrm{m}$. The absorption efficiencies, which in this case are independent of the angle of incidence and polarization, were obtained with analytical Mie theory [23], while the dielectric functions of these three materials were taken from tabulated values (see Appendix A for details). The RHT is characterized here in terms of the room-temperature linear heat conductance, which is normalized in Figs. 1(d)-1(f) by the corresponding black-body result: $G_{\mathrm{BB}}=4 \sigma A F_{12} T^{3}$. In these figures, we present the results computed with Eq. (4) and the absorption efficiencies as well as numerical results for three gaps in the far-field regime $(20 \mu \mathrm{m}, 100 \mu \mathrm{m}$, and $5 \mathrm{~mm})$ that were obtained with the code SCUFF-EM [38,39]. This code implements a fluctuating-surface-current formulation of the RHT problem and provides numerically exact results within the framework of FE theory.

The results of Figs. 1(d)-1(f) show that the far-field RHT between spheres does not overcome the Planckian limit and only becomes comparable to the black-body result in the case of polar dielectrics with a sphere radius of the order of $\lambda_{\mathrm{Th}}$. For small radii, smaller than the corresponding skin depth, the normalized conductance increases linearly with the radius, i.e., the conductance is proportional to the sphere volume because the whole particle contributes to the RHT. In the opposite limit, 

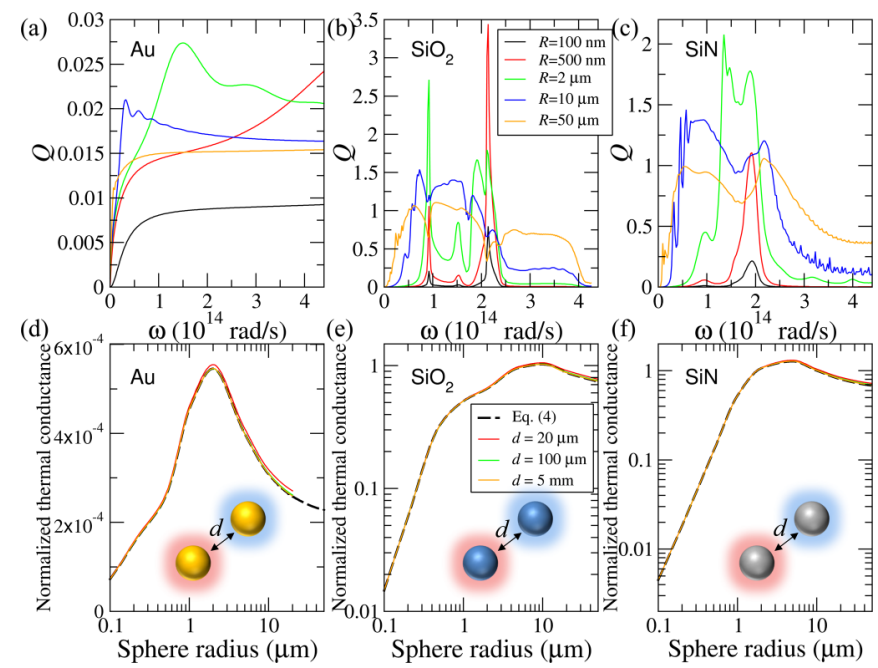

FIG. 1. (a)-(c) Absorption efficiency of a sphere of $\mathrm{Au}$ (a), $\mathrm{SiO}_{2}$ (b), and $\mathrm{SiN}$ (c) as a function of frequency for different values of the sphere radius $R$, see legend in (b). (d)-(f) Room-temperature radiative heat conductance, normalized by the black-body results, for two identical spheres separated by a distance $d$ as a function of the sphere radius. The spheres are made of $\mathrm{Au}(\mathrm{d}), \mathrm{SiO}_{2}(\mathrm{e})$, and $\mathrm{SiN}$ (f). The solid lines are the exact calculations for different gaps, see legend in (e), while the black dashed line is the result obtained with Eq. (4) and the results of (a)-(c).

when the radius is much larger than $\lambda_{\text {Th }}$ the conductance tends to the result for two parallel plates. It is worth stressing that the numerical results obtained for various gaps nicely confirm the validity of Eq. (4) for spheres of arbitrary size and material. In particular, this equation provides very accurate results even for a gap of $20 \mu \mathrm{m}$, which is on the edge of the far-field regime at room temperature.

To further illustrate the difficulty to beat the Planckian limit with optically isotropic objects, we now consider the case of cubes, focusing on polar dielectrics since metals have a very small emissivity (cf. Fig. 1). In Fig. 2, we present the results for the absorption efficiency and the far-field RHT between cubes made of $\mathrm{SiO}_{2}$ and $\mathrm{SiN}$ with sides $L$ ranging from $100 \mathrm{~nm}$ to $20 \mu \mathrm{m}$. The absorption efficiencies, which again refer to a plane wave with normal incidence and the linear polarization indicated in the inset of Fig. 2(b), were obtained numerically with the DDA method [34]. Again, the RHT is characterized here in terms of the room-temperature linear thermal conductance normalized by the corresponding blackbody result. We present in this figure the results computed with Eq. (4), which in this case is equivalent to Eq. (8), and the absorption efficiencies as well as numerical results for two gaps in the far-field regime $(20$ and $500 \mu \mathrm{m})$ that were obtained with the TDDA approach [34]. As one can see, the results of Fig. 2 show that, in general, the far-field RHT between cubes does not overcome the Planckian limit, similar to the case of spheres (Fig. 1). Only when the size of the cubes becomes of the order of $\lambda_{\text {Th }}(\approx 10 \mu \mathrm{m})$ the RHT becomes comparable to the black-body result, and even a bit larger. Notice that the fact that in Figs. 2(c) and 2(e) the thermal conductance is lower for the smallest gap of $20 \mu \mathrm{m}$ is simply due to the fact that the results are normalized by the black-body ones. We emphasize

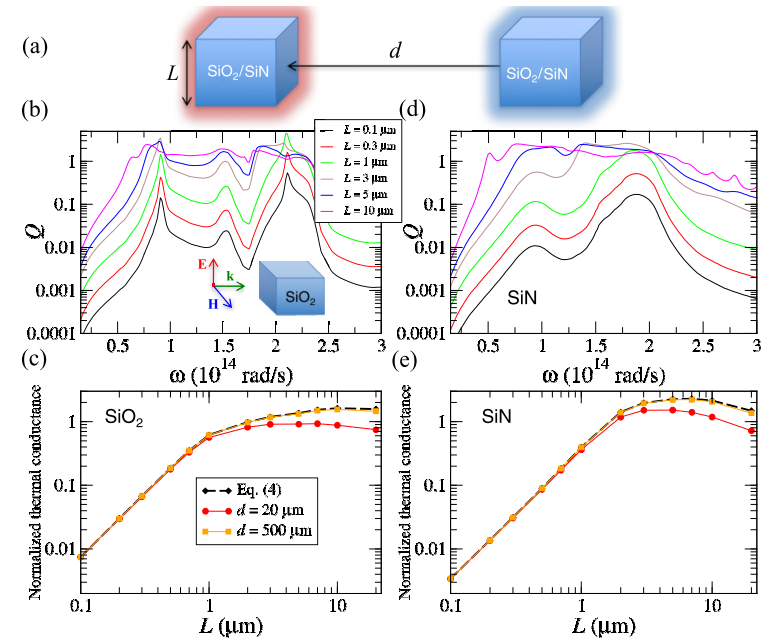

FIG. 2. (a) Two identical cubes made of $\mathrm{SiO}_{2}$ or $\mathrm{SiN}$ with side $L$ and separated by a gap $d$. (b) Absorption efficiency as a function of the frequency for a linearly polarized plane wave impinging with normal incidence in $\mathrm{SiO}_{2}$ cube of side $L$, as indicated in the legend. The inset describes the polarization in terms of the direction of the electric field $\mathbf{E}$, the magnetic field $\mathbf{H}$, and the wave vector $\mathbf{k}$. (c) Room-temperature radiative heat conductance, normalized by the black-body results, for the system shown in (a) as a function of the cube side $L$. The solid lines correspond to the exact calculations for two gaps (see legend), while the black dashed line corresponds to the results obtained by combining Eq. (4) with the results of (b). (d)-(e) The same as in panels (b) and (c), but for $\mathrm{SiN}$.

that in the far-field regime, the thermal conductance decays monotonically with the distance between the objects. It is also worth stressing that once again the numerical results obtained for the largest gap $(500 \mu \mathrm{m})$ nicely coincide with those based on Eq. (4), confirming its validity.

The results presented in this section for spheres and cubes show that although the emissivity of an object can be larger than 1 for some frequencies, this does not imply super-Planckian far-field RHT. From Eqs. (4) and (8), it is obvious that to overcome the Planckian limit one needs thermal emitters with directional emissivities larger than 1 over a broad range of frequencies around the maximum of Planck's distribution, something that is highly nontrivial to achieve. In the following section, we shall show how one can get around this problem.

\section{OVERCOMING THE PLANCKIAN LIMIT}

In the previous section, we have seen the difficulty to overcome the Planckian limit in the far-field regime with optically isotropic bodies and we have also discussed with the help of Eq. (4) why this is so. Fortunately, the same equation also provides a possible strategy to overcome the Planckian limit, which consists in increasing as much as possible the corresponding directional absorption efficiency. This can be done by enhancing the absorption cross section, while maintaining the geometrical one. We shall illustrate this idea in this section by studying the RHT between two parallelepipeds.

Figure 3 shows the far-field RHT between two parallelepipeds of $\mathrm{SiO}_{2}$ and $\mathrm{SiN}$ as well as the relevant emissivities. 


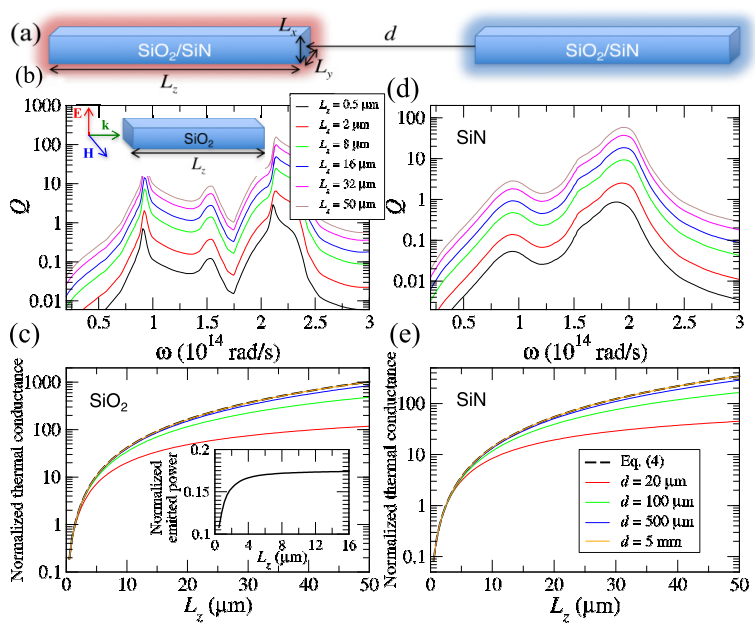

FIG. 3. (a) Two identical parallelepipeds of $\mathrm{SiO}_{2}$ or $\mathrm{SiN}$ with dimensions $L_{x}=L_{y}=0.5 \mu \mathrm{m}$ and varying $L_{z}$ and separated by a gap $d$. (b) Absorption efficiency as a function of frequency for a linearly polarized plane wave impinging with normal incidence, see inset, in a $\mathrm{SiO}_{2}$ parallelepiped with $L_{x}=L_{y}=0.5 \mu \mathrm{m}$ and various values of $L_{z}$, see legend. (c) Room-temperature radiative heat conductance, normalized by the black-body results, for the system of (a) as a function of $L_{z}$. The solid lines are the exact calculations for different gaps, see legend in (e), while the black dashed line is the result obtained with Eq. (4). The inset shows the total power emitted, normalized by the black-body result, by a single $\mathrm{SiO}_{2}$ parallelepiped with $L_{x}=L_{y}=0.5 \mu \mathrm{m}$ and varying $L_{z}$ at $300 \mathrm{~K}$. (d)-(e) The same as in (b) and (c), but for $\mathrm{SiN}$.

It is worth stressing that the RHT in the extreme far-field regime is still given by Eq. (4) where the emissivity is given by the absorption efficiency related to the direction joining the parallelepipeds. In this case, we start with a cube of side $0.5 \mu \mathrm{m}$ and we form an elongated rectangular parallelepiped by progressively changing one of the dimensions, $L_{z}$, while keeping constant the other two, $L_{x}$ and $L_{y}$, see Fig. 3(a). Thus we keep the geometrical cross section constant, while the normal-incidence absorption efficiency is expected to increase linearly with $L_{z}$ as long as it is small compared to the propagation length of the radiation inside the structure, which is hundreds of microns (see next section). This can be understood as follows. The efficiency $Q$ for normal incidence is roughly proportional to $\left[1-\exp \left(-2 \alpha L_{z}\right)\right]$, where $\alpha$ is the attenuation constant of the parallelepiped guided modes, i.e., $1 / 2 \alpha$ is the corresponding propagation length. Thus, in the limit $\alpha L_{z} \ll 1$, $Q \propto L_{z}$. Our calculations of these emissivities, which were performed with the TDDA approach [34], indeed, confirm that they grow linearly with $L_{z}$ in the regime explored here where $\alpha L_{z} \ll 1$, and that they can become much larger than 1 in a broad frequency range, see Figs. 3(b) and 3(d). As shown in Figs. 3(c) and 3(e), this fact leads to a far-field conductance that grows as $L_{z}^{2}$ and it reaches values that overcome the Planckian limit by several orders of magnitude when $L_{z}$ becomes of the order of $50 \mu \mathrm{m}$. Notice that the numerical results for several gaps in the far-field regime coincide with those obtained via Eq. (4) in the limit of sufficiently large gaps. In any case, the Planckian limit is greatly overcome for all gaps in the far-field regime. Let us also stress that these parallelepipeds
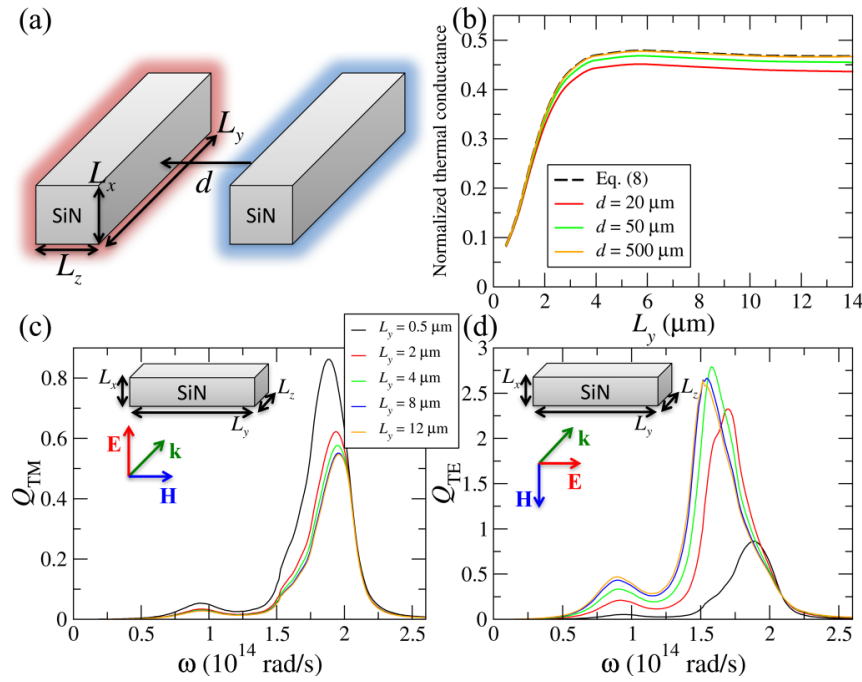

FIG. 4. (a) SiN parallelepipeds with dimensions $L_{x} \times L_{y} \times L_{z}$ separated by a gap $d$. We keep constant the dimensions $L_{x}=$ $L_{z}=0.5 \mu \mathrm{m}$, while we vary the transverse dimension $L_{y}$. (b) The room-temperature radiative thermal conductance normalized by the black-body result for the system shown in (a) as a function of the dimension $L_{y}$. The solid lines correspond to the exact calculations for three gaps in the far-field (see legend), while the black dashed line corresponds to the results obtained by combining Eq. (8) with the results of (c) and (d). (c) Absorption efficiency as a function of the frequency for a plane wave impinging with normal incidence and transverse magnetic (TM) polarization in a $\mathrm{SiN}$ parallelepiped with dimensions $L_{x}=L_{z}=0.5 \mu \mathrm{m}$ and various values of $L_{y}$, as indicated in the legend. The meaning of this polarization is explained in the inset. (d) The same as in (c), but for a transverse electric (TE) polarization.

are not super-Planckian thermal emitters, i.e., they emit less thermal radiation than a corresponding black-body. This is shown in the inset of Fig. 3(c) where we display the total power emitted, normalized by the black-body result, by a single $\mathrm{SiO}_{2}$ parallelepiped with $L_{x}=L_{y}=0.5 \mu \mathrm{m}$ and varying $L_{z}$ at room temperature. This emitted power was computed with the TDDA approach of Ref. [34]. This latter result shows that the super-Planckian RHT found here is possible due to the highly directional thermal emission of these systems.

To further illustrate the crucial role played by the directionality, we now investigate the same parallelepipeds, but oriented as shown in Fig. 4(a). In this case, the parallelepipeds have dimensions $L_{x} \times L_{y} \times L_{z}$, where $z$ is the direction of the line joining the two objects and we keep constant the dimensions $L_{x}=L_{z}=0.5 \mu \mathrm{m}$, while we vary the transverse dimension $L_{y}$. Figures 4(b)-4(d) show the results for the radiative thermal conductance at $300 \mathrm{~K}$ and the relevant absorption efficiencies for these parallelepipeds made of SiN. The corresponding results for $\mathrm{SiO}_{2}$ parallelepipeds are shown in Appendix B. The results for the thermal conductance for various gaps, see Fig. 4(b), were computed with the TDDA approach [34] and the asymptotic result for very large gaps was obtained with the help of Eq. (8) and the absorption efficiencies shown in Figs. 4(c) and 4(d). These efficiencies were calculated with the DDA method [34] and they correspond to a plane wave impinging in a parallelepiped along normal incidence with polarizations 

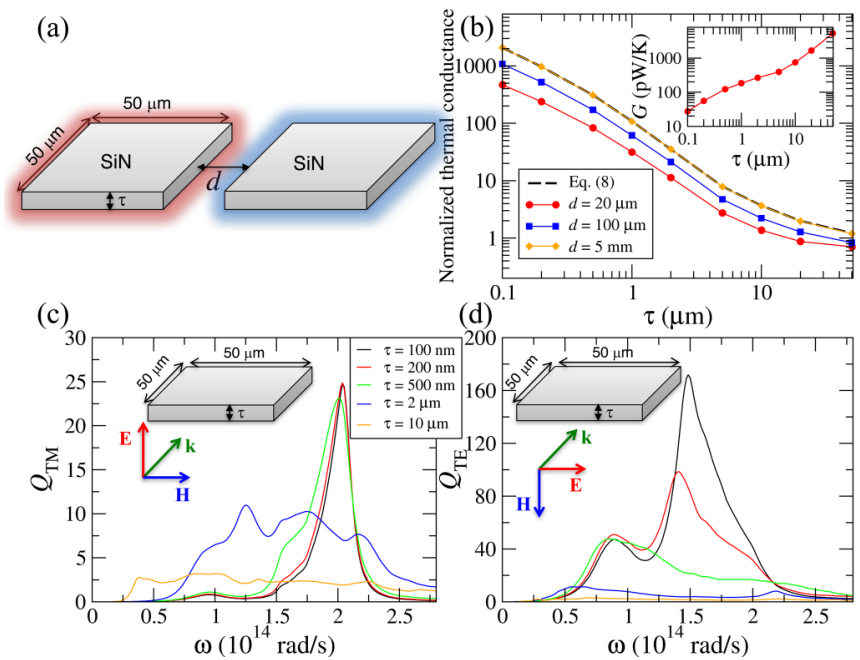

FIG. 5. (a) SiN pads with lateral dimensions of $50 \times 50 \mu \mathrm{m}^{2}$, a thickness $\tau$, and separated by a gap $d$. (b) Room-temperature radiative heat conductance, normalized by the black-body results, for the system of (a) as a function of $\tau$. The solid lines are the exact calculations for three gaps (see legend) and the black dashed line is the result obtained with Eq. (8) and the results of (c) and (d). The inset shows the results for $d=20 \mu \mathrm{m}$ without normalization. (c) Absorption efficiency as a function of frequency for a plane wave impinging with normal incidence and transverse magnetic (TM) polarization in a SiN pad with lateral dimensions of $50 \times 50 \mu \mathrm{m}^{2}$ and various thicknesses, see legend. The inset describes this polarization. (d) The same as in panel (c), but for a transverse electric (TE) polarization.

$x$ or transverse magnetic (TM) polarization, Fig. 4(c), and $y$ or transverse electric (TE) polarization, Fig. 4(d).

The results for the thermal conductance of Fig. 4(b) show that as the transverse dimension $L_{y}$ increases, the RHT remains below the black-body result for all gaps in the far-field regime and the normalized conductance tends to saturate when this dimension becomes on the order of $10 \mu \mathrm{m}$. These results show once more the difficulty to achieve super-Planckian far-field RHT and the crucial role played by the directionality of the thermal emission. On the other hand, it is worth stressing that the numerical results for a large gap of $500 \mu \mathrm{m}$ shown in Fig. 4(b) nicely coincide with the asymptotic result obtained via Eq. (8), which confirms the validity of this result for parallelepipeds.

\section{SUPER-PLANCKIAN FAR-FIELD RADIATIVE HEAT TRANSFER IN SUSPENDED PADS}

The results shown in Fig. 3 clearly demonstrate the possibility to largely overcome the Planckian limit in the far-field regime, but the conductance values for these structures make their measurement challenging. To illustrate super-Planckian heat transfer in a system that can be tested with existing technology, we focus in this section on the analysis of the RHT between two identical SiN pads with fixed lateral dimensions of $50 \times 50 \mu \mathrm{m}^{2}$, see Fig. 5(a), which are larger than $\lambda_{\mathrm{Th}}$ at room temperature, and we vary their thickness, $\tau$, from values much smaller than $\lambda_{\mathrm{Th}}$ to values larger than this wavelength. This challenging system for the theory is inspired by the suspended-pad microdevices that are widely employed for measuring the thermophysical properties of low-dimensional nanostructures (nanotubes, nanowires, or nanoribbons) [2831]. These devices consist of two adjacent SiN membranes suspended by long beams. Each membrane features a platinum resistance heater/thermometer that is normally used to measure the heat conduction through a sample that bridges the gap between the membranes, but they can also be used to measure the RHT across the gap. In recent years, these microdevices have reached sensitivities of $\sim 1 \mathrm{pW} / \mathrm{K}$ and below $[32,33]$.

The RHT between these suspended pads in the extreme farfield regime is described by Eq. (8). The directional absorption efficiencies entering this equation are now those for a plane wave with normal incidence and transverse magnetic (TM) or transverse electric (TE) polarization $\left(Q_{\mathrm{TM}, \mathrm{TE}}(\omega)\right)$, see insets of Figs. 5(c) and 5(d). The results obtained with COMSOL MULTIPHYSICS for these efficiencies for thicknesses varying from $100 \mathrm{~nm}$ to $50 \mu \mathrm{m}$ are displayed in Figs. 5(c) and 5(d) and they show that when $\tau \ll \lambda_{\mathrm{Th}}$ they reach values of up to several hundreds, especially for TE polarization. Using these results together with Eq. (8), we have computed the radiative thermal conductance for different values of $\tau$, see Fig. 5(b). The ratio with the black-body result increases monotonically as $\tau$ decreases, becoming clearly larger than 1 when $\tau<$ $\lambda_{\mathrm{Th}}$, and reaching values as large as 2000 for a thickness of $100 \mathrm{~nm}$. The occurrence of super-Planckian far-field RHT is confirmed by our SCUFF-EM results for several gap sizes, as shown in Fig. 5(b). These results show that, irrespective of the gap, the super-Planckian RHT takes place when $\tau<\lambda_{\text {Th }}$ and that it should be readily observable in suspended-pad microdevices, as we illustrate in the inset of Fig. 5(b) where we show the radiative thermal conductance without normalization. These results also prove once more that Eq. (8) provides the asymptotic result for gaps much larger than the dimensions of the objects, which is when the largest enhancements over the black-body theory occur. Let us also say that we find similar results for the case of $\mathrm{SiO}_{2}$ pads, as we show in Appendix B.

The super-Planckian RHT found for the pads can be further enhanced by increasing their depth, in the very same way as we did for the parallelepipeds. We illustrate this fact in Fig. 6 where we investigate the RHT between two 100-nm-thick SiN pads that are assumed to be infinitely wide and have a depth $L_{z}$ that can be varied, see Fig. 6(a). We have computed with COMSOL MULTIPHYSICS the relevant absorption efficiencies for different values of $L_{z}$ ranging from $100 \mathrm{~nm}$ to $1 \mathrm{~mm}$ and the results are shown in Figs. 6(c) and 6(d). As one can see, the efficiencies grow linearly with $L_{z}$ and reach enormous values in a broad range of frequencies. We have then used these results together with Eq. (8) to compute the far-field RHT in the asymptotic limit of a gap much larger than the pad length. The corresponding results are displayed in Fig. 6(b) and, as one can see, the enhancement over the black-body result now reaches impressive values of the order of $10^{5}$ when $L_{z}$ is of the order of $1 \mathrm{~mm}$.

It is important to stress that none of the systems considered in this work is a super-Planckian emitter, i.e., an object that emits more than a black body with the same dimensions. The existence of super-Planckian emitters, which is in principle 


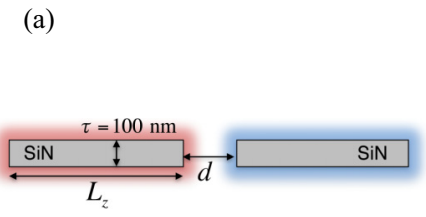

(c)
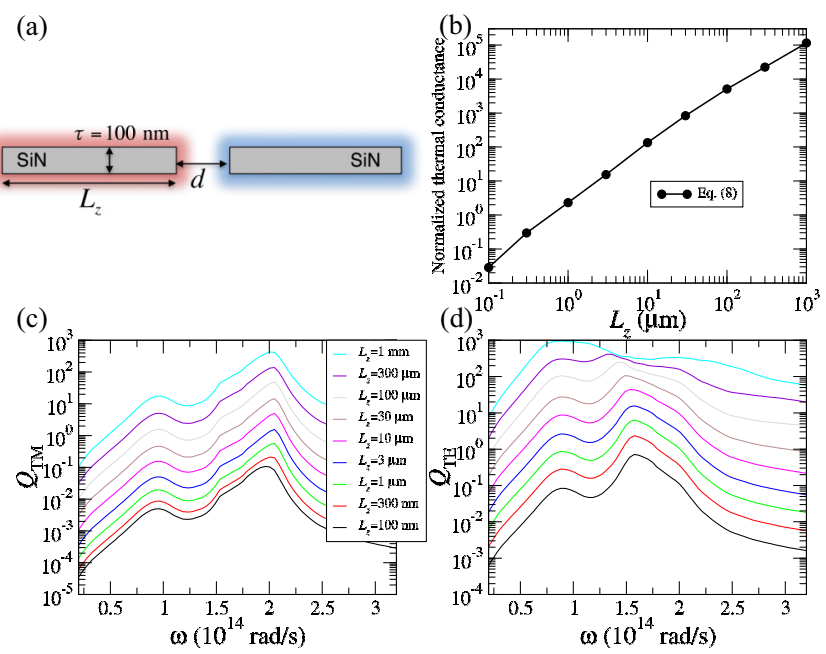

(d)

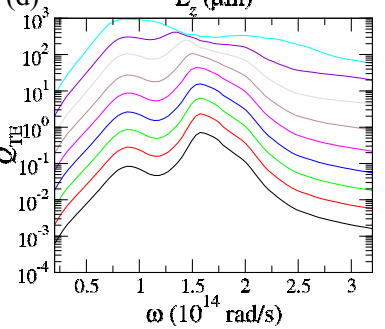

FIG. 6. (a) $\mathrm{SiN}$ pads with a thickness of $100 \mathrm{~nm}$, infinitely wide, a variable depth equal to $L_{z}$ and separated by a gap $d$. (b) Room-temperature radiative heat conductance, normalized by the black-body results, for the system shown in (a) as a function of the pad depth $L_{z}$. The result corresponds to a gap much larger than the pad depth and it was computed with Eq. (8) and the results of (c) and (d). (c) Absorption efficiency as a function of the frequency for a plane wave impinging with normal incidence and transverse magnetic (TM) polarization in a SiN slab with dimensions described in (a). The different curves correspond to different values of $L_{z}$, as indicated in the legend. (d) The same as in (c), but for a transverse electric (TE) polarization.

possible, would make trivial the realization of super-Planckian RHT, but as explained in Introduction, the experimental realization of super-Planckian emitters is extremely challenging. We have already shown in Sec. IV that the parallelepipeds investigated in this work are not super-Planckian emitters, see inset in Fig. 3(c). For completeness, we show in Fig. 7 that the SiN pads studied above, see Fig. 5, are not super-Planckian

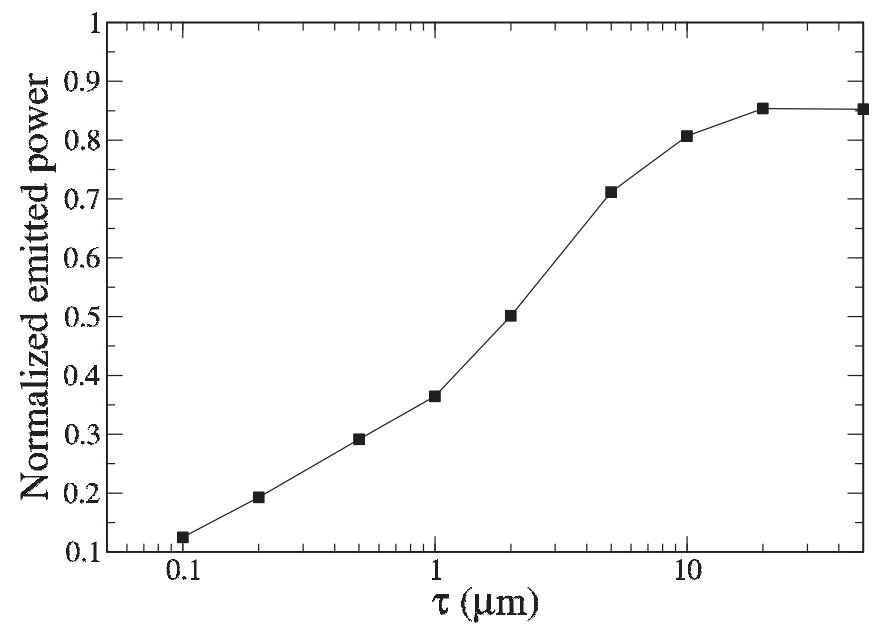

FIG. 7. Total emitted power at room temperature, normalized by the black-body result, by a single $\mathrm{SiN}$ pad with lateral dimensions of $50 \times 50 \mu \mathrm{m}^{2}$ as a function of its thickness $\tau$. Notice that the result is clearly below 1 , which shows that these pads are not super-Planckian emitters. (a)

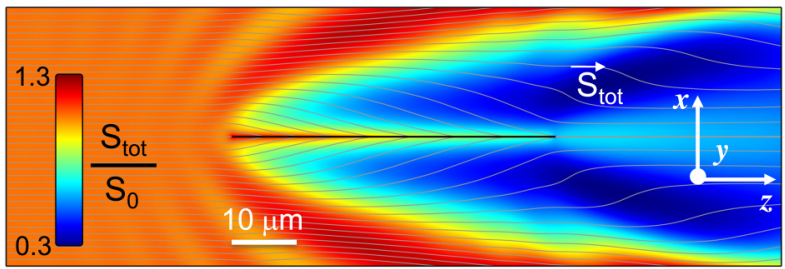

(b)
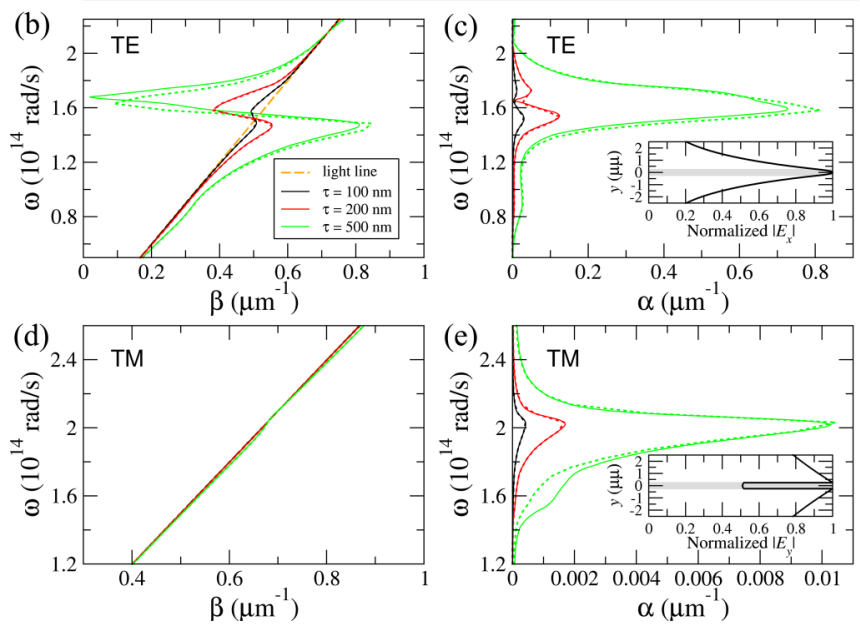

FIG. 8. (a) Magnitude of the total Poynting vector $S_{\text {tot }}$, normalized by the incident value $S_{0}$, in a simulation of a TE-polarized plane wave impinging in a 100-nm-thick SiN pad (in black) for $\omega=1.5 \times 10^{14}$ $\mathrm{rad} / \mathrm{s}$. The pad length is $50 \mu \mathrm{m}$ and it is infinite in the transverse direction. A streamline representation of the Poynting vector is shown in grey solid lines. (b) and (c) Frequency vs propagation constant $\beta$ (b) and attenuation constant $\alpha$ (c) for the TE modes of an infinite SiN slab for different values of its thickness $\tau$. The solid lines correspond to the exact numerical solution of Eq. (11), while the dotted lines correspond to the analytical result of Eqs. (13) and (14). The dashed orange line in (b) is the light line in vacuum, $\omega=\beta c$, and it separates the modes into guided ones on the right-hand side of this line and leaky ones on the left. (d) and (e) The same as in (b) and (c) for TM modes. The insets in (c) and (e) show the normalized electric field profile for the components $E_{x}$ and $E_{y}$, respectively, for a 500-nm-thick slab. The shaded area represents the $\mathrm{SiN}$ slab.

emitters either. In particular, we show in this figure the total power emitted by the $\mathrm{SiN}$ pads considered before (Fig. 5), normalized by the black-body result. This quantity is the total emissivity of this object (integrated in frequencies, angles and polarizations). As one can see in Fig. 7, this emissivity is clearly smaller than 1, irrespective of the thickness, and it tends to its bulk value when the thickness becomes larger than the thermal wavelength.

The last thing that remains to be understood in relation to the RHT between these suspended pads is the origin of their remarkable absorption efficiency. In what follows, we shall show that, in simple terms, these pads behave as (lossy) dielectric waveguides that efficiently absorb the radiation via the excitation of guided modes. This excitation is illustrated in the COMSOL MULTIPHYSICS simulation of Fig. 8(a). In that figure, we display the Poynting vector obtained in a simulation of a TE-polarized plane wave impinging in a $100 \mathrm{~nm}$-thick $\mathrm{SiN}$ pad for a frequency $\omega=1.5 \times 10^{14} \mathrm{rad} / \mathrm{s}$. In this case, the pad length is $50 \mu \mathrm{m}$ and it was assumed to be infinite in the transverse direction. As one can see, the pad is able to 
alter the incoming field over a region that is more than 100 times larger than its thickness. What happens is that, due to the low-impedance mismatch, the incident radiation couples efficiently into a guiding mode that has a large lateral extension outside the pad and the mode is then absorbed during its propagation, with the energy slowly flowing into the pad. The large spatial extent of the guided mode, and its large overlap with the incoming plane wave, is actually responsible for the corresponding cross section becoming so much larger than the physical size of the pad. To understand in more detail how the energy absorption in these pads takes place, we now proceed to study the nature of the guided modes in these dielectric structures.

In order to determine the electromagnetic modes sustained by the pads, we consider an infinite planar waveguide made of a SiN slab of thickness $\tau$ surrounded by air. Our goal is now to obtain the dispersion relation of the guided modes in this structure. Using standard theory of dielectric waveguides [40], one can show that those dispersion relations are given by the solution of the following secular equations:

$$
1-r_{p}^{2} e^{2 i q_{\mathrm{SiN}} \tau}=0
$$

where the index $p$ indicates the polarization of the mode (TE or TM). In this case, the modes with TE polarization have field components $E_{x}, H_{y}$, and $H_{z}$ according to the coordinate system shown in Fig. 8(a), while the TM modes have the following nonvanishing field components: $H_{x}, E_{y}$, and $E_{z}$. On the other hand, the $r_{p}$ 's in Eq. (11) correspond to the Fresnel coefficients

$$
r_{\mathrm{TE}}=\frac{q_{\mathrm{air}}-q_{\mathrm{SiN}}}{q_{\mathrm{air}}+q_{\mathrm{SiN}}} \text { and } r_{\mathrm{TM}}=\frac{\epsilon_{\mathrm{SiN}} q_{\mathrm{air}}-q_{\mathrm{SiN}}}{\epsilon_{\mathrm{SiN}} q_{\mathrm{air}}+q_{\mathrm{SiN}}},
$$

where $q_{j}=\sqrt{\epsilon_{j} \omega^{2} / c^{2}-k^{2}}$ is the transverse component $(y$ component) of the wave vector in medium $j(j=$ air, $\operatorname{SiN})$, $\epsilon_{j}(\omega)$ is the corresponding dielectric function, and $k=\beta+i \alpha$ is the parallel component of the wave vector ( $z$ component). Here, $\beta$ is the propagation constant of the electromagnetic mode and $\alpha$ is the corresponding attenuation constant, which determines both the absorption and the propagation length of the mode.

In Figs. 8(b)-8(e), we present the results for the frequency dependence of the propagation and attenuation constants of the modes sustained by a SiN slab of several thicknesses. In particular, we show with solid lines the exact results obtained with the numerical solution of Eq. (11) for both polarizations (TE or TM). The first thing to notice is that the attenuation constant, which determines the power absorption, strongly depends on the polarization and exhibits much larger values for the TE case, which explains the larger efficiency for this polarization, see Fig. 5(d). Moreover, the frequency dependence of the attenuation constant reproduces the corresponding dependence of the absorption efficiencies for both polarizations in the limit of thin pads. Interestingly, there is a frequency range in which the slab behaves as a hollow dielectric waveguide [40], with the real part of the core dielectric function being smaller than that of the cladding, and the propagation for TE polarization occurs via leaky modes [40], see Figs. 8(b) and $8(\mathrm{c})$.
It is worth recalling that these planar dielectric waveguides have no frequency cut-off, i.e., there exists at least one electromagnetic guiding mode for TE and TM polarizations for any value of the thickness $\tau$. In order to have a second mode in our slab waveguide, $q_{\mathrm{SiN}}$ must fulfill the condition $\operatorname{Re}\left\{q_{\mathrm{SiN}}\right\} \tau=\pi$. In our case, the thinnest slab at which there is an additional mode for a certain $\omega$ is $\tau=1.8 \mu \mathrm{m}$, for both TE and TM polarization. Thus, for the thickness range analyzed in Fig. 8, there is only one electromagnetic guided mode in the SiN slab.

In the limit of very thin slabs $\left(q_{\mathrm{SiN}} \tau \ll 1\right)$, it is possible to find an analytical solution of Eq. (11). In this limit, the mode dispersion relations are given by

$$
\begin{aligned}
& k_{\mathrm{TE}}=\frac{\omega}{c} \sqrt{1+\left(\frac{\omega \tau\left(\epsilon_{\mathrm{SiN}}-1\right)}{2 c}\right)^{2}}, \\
& k_{\mathrm{TM}}=\frac{\omega}{c} \sqrt{1+\left(\frac{\omega \tau\left(\epsilon_{\mathrm{SiN}}-1\right)}{2 c \epsilon_{\mathrm{SiN}}}\right)^{2}} .
\end{aligned}
$$

These expressions provide a very good approximation for the dispersion relation of the modes for the thickness range analyzed, as the dashed lines of Figs. 8(b)-8(e) show. In the case of very thin slabs, it is possible to further simplify Eqs. (13) and (14) to obtain the following analytical expressions for the attenuation constant for both polarizations:

$$
\begin{gathered}
\alpha_{\mathrm{TE}}(\omega)=\frac{\omega^{3} \tau^{2}}{4 c^{3}} \operatorname{Im}\left\{\epsilon_{\mathrm{SiN}}(\omega)\right\}\left[\operatorname{Re}\left\{\epsilon_{\mathrm{SiN}}(\omega)\right\}-1\right] \\
\alpha_{\mathrm{TM}}(\omega)=\frac{\omega^{3} \tau^{2}}{4 c^{3}} \frac{\operatorname{Im}\left\{\epsilon_{\mathrm{SiN}}(\omega)\right\}\left[\left|\epsilon_{\mathrm{SiN}}(\omega)\right|^{2}-\operatorname{Re}\left\{\epsilon_{\mathrm{SiN}}(\omega)\right\}\right]}{\left|\epsilon_{\mathrm{SiN}}(\omega)\right|^{4}}
\end{gathered}
$$

These expressions show that the attenuation constant that determines the power absorption in the slab scales with the thickness as $\tau^{2}$, i.e., the propagation length of these modes increases upon reducing the waveguide thickness. It is important to emphasize that this propagation length of these modes, which is given by $1 / 2 \alpha$, reaches values even larger than $1 \mathrm{~mm}$ for thicknesses below a few hundreds of $\mathrm{nm}$ for the relevant frequencies. This is especially true for the TM polarization. This fact explains why in all the cases considered in this section, see Figs. 5 and 6, the absorption efficiencies grow linearly with the dimension $L_{z}$. The idea is that, as we explained in Sec. IV, the efficiencies $Q$ for normal incidence are proportional to the factor $\left[1-\exp \left(-2 \alpha L_{z}\right)\right]$, and since we have investigated here the limit $\alpha L_{z} \ll 1$, then $Q \propto L_{z}$.

Finally, and in order to get some further insight into the nature of the electromagnetic modes supported by these $\mathrm{SiN}$ slabs, we have plotted the electric field component that is perpendicular to the propagation direction of the mode $\left(E_{x}\right.$ for TE polarization and $E_{y}$ for the TM case), see insets of Figs. 8(c) and 8(e). Both electric profiles are represented as a function of the $y$ coordinate for a slab of thickness $\tau=500 \mathrm{~nm}$ and frequencies $\omega=1.47 \times 10^{14} \mathrm{rad} / \mathrm{s}$ and $\omega=1.98 \times 10^{14}$ $\mathrm{rad} / \mathrm{s}$ for the TE and TM mode, respectively. The $E_{y}$ profile of the TM mode is discontinuous at the boundary and it extends longer in the $y$ direction. Therefore it has a smaller amplitude inside the slab as compared to the $E_{x}$ profile of the TE mode. This explains why the attenuation constant is smaller in the 
TM case, and therefore the absorption along the SiN pads is larger for this polarization.

\section{CONCLUSIONS}

In summary, we have presented in this work a theoretical study that demonstrates that the far-field RHT between objects with some of their dimensions smaller than the thermal wavelength can greatly overcome the Planckian limit. By finding a connection between the far-field RHT and the directional absorption efficiencies of the objects involved, we have provided very intuitive guidelines on how to achieve super-Planckian far-field RHT. In particular, we have shown that a huge super-Planckian far-field RHT can be achieved in micrometer-sized suspended devices of great importance for the field of thermal transport [28-33], which in turn can be used to quantitatively test our predictions.

From a more general perspective, our work illustrates the need to revisit the far-field RHT between micro- and nanosystems in the light of fluctuational electrodynamics theory. Our work is also important for the study of thermalization of small objects [27] with implications, e.g., in cavity optomechanics experiments [41] or in the study of interstellar dust in astrophysics [42]. Finally, our work also raises the question of the ultimate limit of the super-Planckian far-field RHT in low-dimensional systems such as nanowires and 2D materials.

\section{ACKNOWLEDGMENTS}

We are indebted to P. Reddy, E. Meyhofer, and D. Thompson for drawing attention to this problem and for sharing with us their experimental results prior to publication. We also thank A. García-Martín, E. Moreno, and Y. Shi for fruitful discussions. We acknowledge funding from the Spanish MINECO (FIS2015-64951-R, MAT2014-53432C5-5-R, FIS2014-53488-P, FIS2017-84057-P), the Comunidad de Madrid (S2013/MIT-2740), the European Union Seventh Framework Programme (FP7-PEOPLE-2013-CIG630996, FP7-PEOPLE-2013-CIG-618229), and the European Research Council (ERC-2011-AdG-290981 and ERC-2016STG-714870). V.F.-H. acknowledges support from "la Caixa" Foundation and J.C.C. thanks the DFG and SFB767 for sponsoring his stay at the University of Konstanz as Mercator Fellow.

\section{APPENDIX A: DIELECTRIC CONSTANTS}

In this appendix, we provide the different dielectric functions that were used for the calculations of both the absorption cross-sections and the radiative heat transfer. We assumed that these dielectric functions only depend on frequency (local approximation) and we took these functions from experimental results reported in the literature. To be precise, we took the dielectric functions of Au from Ref. [43], the $\mathrm{SiO}_{2}$ function was taken from Palik [44], and that of $\mathrm{SiN}$ from Cataldo et al. [45]. These three dielectric functions are shown in Fig. 9 for the frequency range relevant for our calculations.

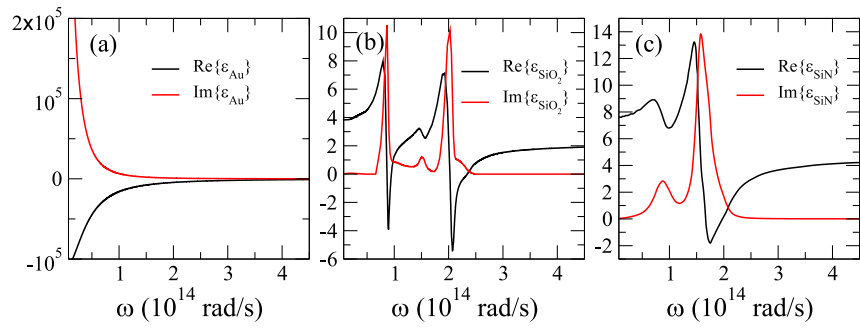

FIG. 9. (a)-(c) Real and imaginary parts of the dielectric functions employed in our calculations as a function of frequency for $\mathrm{Au}$, $\mathrm{SiO}_{2}$, and $\mathrm{SiN}$.

\section{APPENDIX B: Additional results for $\mathrm{SiO}_{2}$ STRUCTURES}

In this appendix, we briefly present additional results for the RHT of several structures that were analyzed in the main text for the case of $\mathrm{SiN}$, but this time for $\mathrm{SiO}_{2}$ bodies. First of all, we present in Fig. 10 the RHT results for the parallelepipeds of Fig. 4, but in this case, made of $\mathrm{SiO}_{2}$. These results confirm the basic conclusions drawn from Fig. 4 for $\mathrm{SiN}$, the main difference being that the normalized thermal conductance adopts lower values than in the $\mathrm{SiN}$ case as $L_{y}$ increases.

In Fig. 11, we show the results for the far-field radiative thermal conductance and the corresponding absorption efficiencies for $\mathrm{SiO}_{2}$ pads with fixed lateral dimensions of $50 \times 50 \mu \mathrm{m}^{2}$, see Fig. 11(a), and varying thickness, $\tau$. This is the system investigated in Fig. 5 of the main text, but now for $\mathrm{SiO}_{2}$. The results for $\mathrm{SiO}_{2}$ are indeed very similar to those for SiN and confirm the possibility to greatly overcome the Planckian limit with polar dielectrics when the pad thickness is smaller than the thermal wavelength. As in the case of the SiN pads, the absorption efficiencies were computed with COMSOL MULTIPHYSICS, while the numerical simulation of the RHT were carried out with SCUFF-EM code.
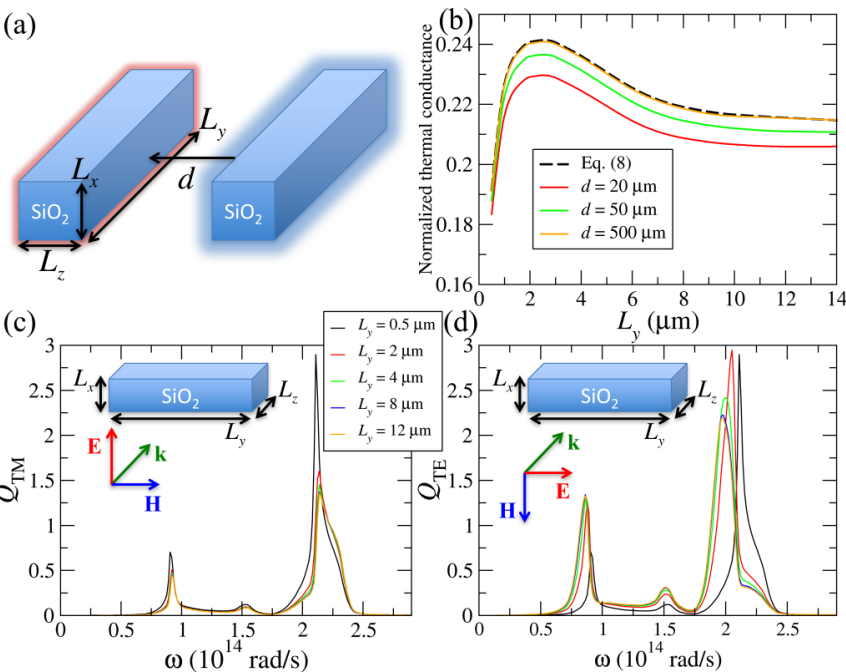

FIG. 10. The same as in Fig. 4, but for $\mathrm{SiO}_{2}$ parallelepipeds. 


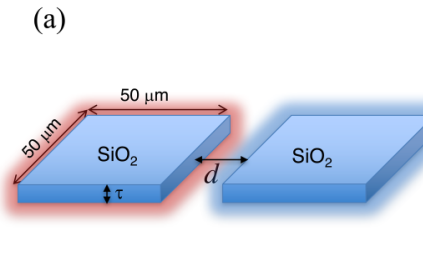

(c)
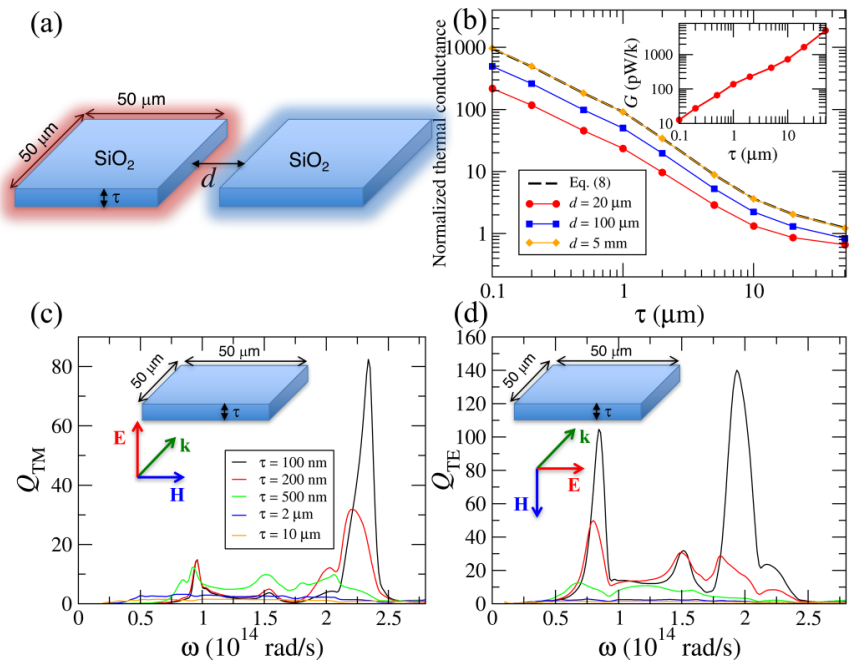

FIG. 11. The same as in Fig. 5, but for $\mathrm{SiO}_{2}$ pads.

\section{APPENDIX C: VIEW FACTORS}

In most of the cases discussed in this work, we have presented the results of the far-field radiative thermal conductance normalized by the corresponding black-body results. Those results are given by the Stefan-Boltzmann law: $G_{\mathrm{BB}}=$ $4 \sigma A F_{12} T^{3}$, where $\sigma$ is the Stefan-Boltzmann constant, $A$ is the area of the bodies (assumed to be identical), $T$ is the absolute temperature, and $F_{12}$ is the so-called view factor [1]. For completeness, we present in this appendix the expressions for the different view factors used in this work. Moreover, let us recall that these view factors also appear in the formulas for the far-field RHT, see, e.g., Eqs. (4) and (8).
The view factor between two aligned parallel rectangles of sides $L_{x}$ and $L_{y}$ and separated by a gap $d$ is given by [1]

$$
\begin{aligned}
F_{12}^{\text {rectangles }}= & \frac{2}{\pi \tilde{L}_{x} \tilde{L}_{y}}\left\{\ln \left[\frac{\left(1+\tilde{L}_{x}^{2}\right)\left(1+\tilde{L}_{y}^{2}\right)}{1+\tilde{L}_{x}^{2}+\tilde{L}_{y}^{2}}\right]^{1 / 2}\right. \\
& +\tilde{L}_{x}\left(1+\tilde{L}_{y}^{2}\right)^{1 / 2} \arctan \left[\frac{\tilde{L}_{x}}{\left(1+\tilde{L}_{y}^{2}\right)^{1 / 2}}\right] \\
& +\tilde{L}_{y}\left(1+\tilde{L}_{x}^{2}\right)^{1 / 2} \arctan \left[\frac{\tilde{L}_{y}}{\left(1+\tilde{L}_{x}^{2}\right)^{1 / 2}}\right] \\
& \left.-\tilde{L}_{x} \arctan \left(\tilde{L}_{x}\right)-\tilde{L}_{y} \arctan \left(\tilde{L}_{y}\right)\right\},
\end{aligned}
$$

where $\tilde{L}_{x}=L_{x} / d$ and $\tilde{L}_{y}=L_{y} / d$. This is the view factor that has been used for the calculation of the radiative heat transfer between parallelepipeds, including the pads and cubes. In the two-spheres case, there is no analytical expression for the view factor. However, when the gap $d$ is larger than the sphere radius $R$, it can be approximated by [37]

$$
F_{12}^{\text {spheres }}=\frac{1}{2}\left\{1-\left[1-\left(\frac{R}{d+2 R}\right)^{2}\right]^{1 / 2}\right\} .
$$

This expression yields a value that differs less than $1 \%$ from the exact numerical result in most of the cases explored in this work. For all other cases, we have considered the exact numeric view factor. To be precise, the value of the view factor in those cases is $F_{12}^{\text {spheres }}(d=100 \mu \mathrm{m}, R=50 \mu \mathrm{m})=F_{12}^{\text {spheres }}(d=$ $20 \mu \mathrm{m}, R=10 \mu \mathrm{m})=0.0161, F_{12}^{\text {spheres }}(d=20 \mu \mathrm{m}, R=$ $20 \mu \mathrm{m})=0.0294$, and $F_{12}^{\text {spheres }}(d=20 \mu \mathrm{m}, R=50 \mu \mathrm{m})=$ 0.0485 .
[1] M. F. Modest, Radiative Heat Transfer (Academic Press, New York, 2013).

[2] M. Planck, The Theory of Thermal Radiation (Blakiston, Philadelphia, 1914).

[3] K. Joulain, J.-P. Mulet, F. Marquier, R. Carminati, and J.-J. Greffet, Surface electromagnetic waves thermally excited: radiative heat transfer, coherence properties and casimir forces revisited in the near field, Surf. Sci. Rep. 57, 59 (2005).

[4] S. Basu, Z. M. Zhang, and C. J. Fu, Review of near-field thermal radiation and its application to energy conversion, Int. J. Energy Res. 33, 1203 (2009).

[5] B. Song, A. Fiorino, E. Meyhofer, and P. Reddy, Near-field radiative thermal transport: from theory to experiment, AIP Adv. 5, 053503 (2015).

[6] D. Polder and M. Van Hove, Theory of radiative heat transfer between closely spaced bodies, Phys. Rev. B 4, 3303 (1971).

[7] S. M. Rytov, Y. A. Kravtsov, and V. I. Tatarskii, Principles of Statistical Radiophysics (Springer-Verlag, Heidelberg, 1989), Vol. 3.

[8] A. Kittel, W. Müller-Hirsch, J. Parisi, S.-A. Biehs, D. Reddig, and M. Holthaus, Near-field Heat Transfer in a Scanning Thermal Microscope, Phys. Rev. Lett. 95, 224301 (2005).
[9] E. Rousseau, A. Siria, G. Jourdan, S. Volz, F. Comin, J. Chevrier, and J.-J. Greffet, Radiative heat transfer at the nanoscale, Nat. Photon. 3, 514 (2009).

[10] S. Shen, A. Narayanaswamy, and G. Chen, Surface phonon polaritons mediated energy transfer between nanoscale gaps, Nano Lett. 9, 2909 (2009).

[11] R. S. Ottens, V. Quetschke, S. Wise, A. A. Alemi, R. Lundock, G. Mueller, D. H. Reitze, D. B. Tanner, and B. F. Whiting, Near-Field Radiative Heat Transfer between Macroscopic Planar Surfaces, Phys. Rev. Lett. 107, 014301 (2011).

[12] T. Kralik, P. Hanzelka, M. Zobac, V. Musilova, T. Fort, and M. Horak, Strong Near-Field Enhancement of Radiative Heat Transfer between Metallic Surfaces, Phys. Rev. Lett. 109, 224302 (2012).

[13] P. J. van Zwol, L. Ranno, and J. Chevrier, Tuning Near Field Radiative Heat Flux through Surface Excitations with a Metal Insulator Transition, Phys. Rev. Lett. 108, 234301 (2012).

[14] P. J. van Zwol, S. Thiele, C. Berger, W. A. de Heer, and J. Chevrier, Nanoscale Radiative Heat Flow Due to Surface Plasmons in Graphene and Doped Silicon, Phys. Rev. Lett. 109, 264301 (2012). 
[15] L. Worbes, D. Hellmann, and A. Kittel, Enhanced Near-Field Heat Flow of a Monolayer Dielectric Island, Phys. Rev. Lett. 110, 134302 (2013).

[16] R. St-Gelais, B. Guha, L. X. Zhu, S. H. Fan, and M. Lipson, Demonstration of strong near-field radiative heat transfer between integrated nanostructures, Nano Lett. 14, 6971 (2014).

[17] B. Song, Y. Ganjeh, S. Sadat, D. Thompson, A. Fiorino, V. Fernández-Hurtado, J. Feist, F. J. Garcia-Vidal, J. C. Cuevas, P. Reddy, and E. Meyhofer, Enhancement of near-field radiative heat transfer using polar dielectric thin films, Nat. Nanotechnol. 10, 253 (2015).

[18] K. Kim, B. Song, V. Fernández-Hurtado, W. Lee, W. Jeong, L. Cui, D. Thompson, J. Feist, M. T. H. Reid, F. J. Garcia-Vidal, J. C. Cuevas, E. Meyhofer, and P. Reddy, Radiative heat transfer in the extreme near field, Nature (London) 528, 387 (2015).

[19] R. St-Gelais, L. Zhu, S. H. Fan, and M. Lipson, Near-field radiative heat transfer between parallel structures in the deep subwavelength regime, Nat. Nanotechnol. 11, 515 (2016).

[20] B. Song, D. Thompson, A. Fiorino, Y. Ganjeh, P. Reddy, E. Meyhofer, Radiative heat conductances between dielectric and metallic parallel plates with nanoscale gaps, Nat. Nanotechnol. 11, 509 (2016).

[21] M. P. Bernardi, D. Milovich, and M. Francoeur, Radiative heat transfer exceeding the blackbody limit between macroscale planar surfaces separated by a nanosize vacuum gap, Nat. Commun. 7, 12900 (2016).

[22] S.-A. Biehs and P. Ben-Abdallah, Revisiting super-Planckian thermal emission in the far-field regime, Phys. Rev. B 93, 165405 (2016).

[23] C. F. Bohren and D. R. Huffman, Absorption and scattering of light by small particles (Wiley, New York, 1998).

[24] J. A. Schuller, T. Taubner, and M. L. Brongersma, Optical antenna thermal emitters, Nat. Photon. 3, 658 (2009).

[25] G. W. Kattawar and M. Eisner, Radiation from a homogeneous isothermal sphere, Appl. Opt. 9, 2685 (1970).

[26] V. A. Golyk, M. Krüger, and M. Kardar, Heat radiation from long cylindrical objects, Phys. Rev. E 85, 046603 (2012).

[27] C. Wuttke and A. Rauschenbeutel, Thermalization Via Heat Radiation of an Individual Object Thinner than the Thermal Wavelength, Phys. Rev. Lett. 111, 024301 (2013).

[28] P. Kim, L. Shi, A. Majumdar, and P. L. McEuen, Thermal Transport Measurements of Individual Multiwall Carbon Nanotubes, Phys. Rev. Lett. 87, 215502 (2001).

[29] L. Shi et al. Measuring thermal and thermoelectric properties of one-dimensional nanostructures using a microfabricated device, J. Heat Transfer 125, 881 (2003).
[30] S. Lee et al., Anisotropic in-plane thermal conductivity of black phosphorus nanoribbons at temperatures higher than $100 \mathrm{~K}$, Nat. Commun. 6, 8573 (2015).

[31] S. Lee et al., Anomalously low electronic thermal conductivity in metallic vanadium dioxide, Science 355, 371 (2017).

[32] S. Sadat, E. Meyhofer, and P. Pramod, Resistance thermometrybased picowatt-resolution heat-flow calorimeters, Appl. Phys. Lett. 102, 163110 (2013).

[33] J. Zheng, M. C. Wingert, E. Dechaumphai, and R. Chen, Subpicowatt/kelvin resistive thermometry for probing nanoscale thermal transport, Rev. Sci. Instrum. 84, 114901 (2013).

[34] R. M. Abraham Ekeroth, A. García-Martín, and J. C. Cuevas, Thermal discrete dipole approximation for the description of thermal emission and radiative heat transfer of magneto-optical systems, Phys. Rev. B 95, 235428 (2017).

[35] S. Edalatpour, M. Cuma, T. Trueax, R. Backman, and M. Francoeur, Convergence analysis of the thermal discrete dipole approximation, Phys. Rev. E 91, 063307 (2015).

[36] S. Edalatpour and M. Francoeur, Near-field radiative heat transfer between arbitrarily shaped objects and a surface, Phys. Rev. B 94, 045406 (2016).

[37] N. Juul, Investigation of approximate methods for calculation of the diffuse radiation configuration view factor between two spheres, Lett. Heat Mass Transfer 3, 513 (1976).

[38] A. W. Rodriguez, M. T. H. Reid, and S. G. Johnson, Fluctuatingsurface-current formulation of radiative heat transfer: Theory and applications, Phys. Rev. B 88, 054305 (2013).

[39] M. T. H. Reid and S. G. Johnson, Efficient computation of power, force and torque in BEM scattering calculations, IEEE T. Antenn. Propag. 63, 3588 (2015).

[40] D. Marcuse, Theory of Dielectric Optical Waveguides (Academic Press, London, 1991).

[41] D. E. Chang, C. A. Regal, S. B. Papp, D. J. Wilson, J. Ye, O. Painter, H. J. Kimble, and P. Zoller, Cavity opto-mechanics using an optically levitated nanosphere, Proc. Natl. Acad. Sci. U. S. A. 107, 1005 (2010).

[42] B. T. Draine, Physics of the Interstellar and Intergalactic Medium (Princeton University Press, Princeton, 2011).

[43] R. L. Olmon, B. Slovick, T. W. Johnson, D. Shelton, S.-H. Oh, G. D. Boreman, and M. B. Raschke, Optical dielectric function of gold, Phys. Rev. B 86, 235147 (2012).

[44] E. D. Palik, Handbook of Optical Constants of Solids (Academic Press, London, 1985).

[45] G. Cataldo, J. A. Beall, H.-M. Cho, B. McAndrew, M. D. Niemack, and E. L. Wollack, Infrared dielectric properties of low-stress silicon nitride, Opt. Lett. 37, 4200 (2012). 